\title{
A relação de incerteza de Maccone-Pati
}

\author{
The Maccone-Pati uncertainty relation
}

\author{
Jonas Maziero*1 \\ ${ }^{1}$ Departamento de Física, Centro de Ciências Naturais e Exatas, Universidade Federal de Santa Maria, Avenida Roraima 1000, \\ 97105-900, Santa Maria, RS, Brazil
}

Recebido em 18 de Janeiro de 2017. Revisado em 27 de Abril de 2017. Aceito em 27 de Abril de 2017

\begin{abstract}
A existência de observáveis incompatíveis em mecânica quântica (MQ) é uma das suas características mais notáveis e pode ser revelada e formalizada através de relações de incerteza. A relação de incerteza de HeisenbergRobertson-Schrödinger (RIHRS) foi provada nos primórdios do formalismo quântico e está unipresente tanto no ensino quanto na pesquisa em MQ. Todavia, a RIHRS possui o chamado problema da trivialidade, ou seja, ela não fornece informação alguma sobre a possível incompatibilidade entre dois observáveis se o estado no qual o sistema foi preparado for autovetor de um deles. Este problema foi resolvido recentemente por Lorenzo Maccone e Arun K. Pati, depois de aproximadamente 85 anos de existência da RIHRS. Neste artigo começamos fazendo uma breve discussão sobre aspectos gerais do princípio de incerteza em MQ e recapitulando a prova da RIHRS. Na sequência apresentamos de forma simples a prova da relação de incerteza de Maccone-Pati, que pode ser obtida usando basicamente a lei dos paralelogramos e a desigualdade de Cauchy-Schwarz.
\end{abstract}

Palavras-chave: Mecânica Quântica, relações de incerteza

The existence of incompatible observables constitutes one of the most prominent characteristics of quantum mechanics (QM) and can be revealed and formalized through uncertainty relations. The Heisenberg-RobertsonSchrödinger uncertainty relation (HRSUR) was proved at the dawn of quantum formalism and is ever-present in the teaching and research on QM. Notwithstanding, the HRSUR possess the so called triviality problem. That is to say, the HRSUR yields no information about the possible incompatibility between two observables if the system was prepared in a state which is an eigenvector of one of them. After about 85 years of existence of the HRSUR, this problem was solved recently by Lorenzo Maccone and Arun K. Pati. In this article, we start doing a brief discussion of general aspects of the uncertainty principle in QM and recapitulating the proof of HRSUR. Afterwards we present in simple terms the proof of the Maccone-Pati uncertainty relation, which can be obtained basically via the application of the parallelogram law and the Cauchy-Schwarz inequality.

Keywords: Quantum mechanics, uncertainty relations

\section{Introdução}

Podemos dizer que incerteza é parte de nossas vidas [1]. Contudo, a incerteza com que nos deparamos no dia a dia é frequentemente algo mais nosso como observadores do que uma propriedade intrínseca dos entes físicos com os quais interagimos. Esta situação muda completamente em circunstâncias onde efeitos quânticos são observacionalmente importantes. Para esses sistemas a incerteza é um caráter fundamental, ou seja, simplesmente não podemos, em geral, prever o que vai acontecer no futuro, mesmo quando temos toda informação que podemos ter sobre a história do objeto que estamos analisando [2 6].

Para sistemas cuja descrição requer o uso das regras da Mecânica Quântica (MQ) [7-10], só podemos prever a probabilidade (chance ou frequência relativa) de um evento ocorrer. Esse fato pode ser atribuído à existência, em MQ, de observáveis incompatíveis (OI). Como esses observáveis são representados por matrizes Hermitianas

*Endereço de correspondência: jonas.maziero@ufsm.br que não comutam, e que por conseguinte não podem compartilhar todos os autovetores, podemos, através da medida de um deles, preparar um estado que é uma superposição coerente dos autovetores de outro observável. Nesse caso, a incerteza sobre esse último observável é necessariamente não nula, o que é associado com uma "largura" (medida em geral usando o desvio padrão ou variância) não nula da distribuição de probabilidades (DP) para os seus autovalores.

Se preparamos um sistema físico no estado $|\xi\rangle$ via a medida de um observável $\hat{C}[9]$, podemos utilizar a estrutura cinemática da MQ para obter restrições sobre o quão pequeno pode ser o produto ou soma das incertezas sobre outros dois observáveis $\hat{A}$ e $\hat{B} 11$ 14. Esse tipo de desigualdade, que é chamada de relação de incerteza (RI) de preparação (RIP), depende de $|\xi\rangle$ e dos observáveis considerados e será o tema principal desse artigo.

O objetivo de uma RIP é identificar a incompatibilidade, dependente de estado, de dois observáveis através da geral impossibilidade de prepararmos o sistema físico 
de interesse em um estado para o qual ambas as distribuições de probabilidade (para os autovalores desses observáveis) tenham variância nula. A presença frequente desse tipo de RI nos livros texto de MQ aponta para a sua importância didática no contexto do aprendizado dos fundamentos dessa teoria. Ademais, as RI têm diversas aplicações práticas, indo desde a justificativa para a utilização de um campo escalar complexo em MQ [15] até áreas tais como criptografia quântica [16] e testemunho de emaranhamento quântico 17, 18.

Existem vários outros aspectos sobressalentes do princípio de incerteza da MQ 19], alguns dos quais mencionaremos neste parágrafo. Dentro do contexto da Ciência da Informação Quântica 20], especialmente em Criptografia Quântica [21], RI erro-perturbação são particularmente importantes por fornecerem limites entre a quantidade de informação que podemos extrair através de medidas feitas sobre um sistema e a consequente perturbação que será infringida sobre o seu estado 22 24. Vale mencionar que, como ao medir um observável para extrair informação sobre o sistema em geral modificaremos a DP de outro observável, as RI erro-perturbação também são conhecidas como RI para medidas conjuntas de observáveis. Por outro lado, o reconhecimento de que correlações quânticas, tais como emaranhamento [25,26] e discórdia [27,28], podem ser utilizadas como um recurso para a manipulação mais eficiente de informação, motivou a proposta de RI com memórias quânticas 29.30. Aqui, se mostrou que as limitações nas incertezas sobre OI de um sistema podem ser enfraquecidas se os observadores estiverem quanticamente correlacionados com ele. Ademais, RI entrópicas, independentes de estado, podem ser obtidas que vinculam as "entropias" das DP dos autovalores de OI [31]. Outro tipo importante de RI na MQ são as que envolvem parâmetros que não são representados por operadores Hermitianos, tais com tempo ou fase 32 . Um exemplo desse tipo de RI é a RI energia-tempo, que possui um papel fundamental para o estabelecimento de limites para a rapidez de mudança de estados quânticos, o que pode ser utilizado, por exemplo, para limitar a eficiência de um processador quântico de informação 33 . É curioso observar que, como boa parte das RI mencionadas acima envolve a medida do valor médio do produto de dois OI e $\hat{A} \hat{B}$ não é um operador Hermitiano, elas não são experimentalmente acessíveis [34]. Recentemente um esquema foi proposto para tornar possível a verificação experimental de RI envolvendo o valor médio de $\hat{A} \hat{B} 35,36$, mas tal técnica ainda não foi colocada em prática.

A sequência desse artigo está organizada da seguinte maneira. Na Sec. 2 discutimos a desigualdade de CauchySchwarz e sua aplicação na obtenção da relação de incerteza de Heisenberg, Robertson e Schrödinger (RIHRS). Depois de discutir o problema da trivialidade da RIHRS, provamos, na Sec. 3. a relação de incerteza de Maccone e Pati (RIMP). Em contraste com a RIHRS, a RIMP fornece limites inferiores não nulos para a soma das variâncias de dois observáveis sempre que o estado do sistema não for autovetor simultâneo de ambos os operadores Hermitianos correspondentes; portanto a RIMP pode ser vista como um aperfeiçoamento da RIHRS. Por fim, depois de apresentar um exemplo de aplicação dessas relações de incerteza na $\mathrm{Sec}$. 4. alguns comentários finais são incluídos na Sec. 5

\section{A relação de incerteza de Heisenberg-Robertson-Schrödinger e o problema da trivialidade}

Devido à sua importância para as provas dos resultados que discutiremos neste artigo, iniciaremos recapitulando a desigualdade de Cauchy-Schwarz (DCS). A DCS estabelece que para quaisquer dois vetores não nulos $|\psi\rangle$ e $|\phi\rangle$ em um espaço de Hilbert $\mathcal{H}[9]$, temos que

$$
\langle\psi \mid \psi\rangle\langle\phi \mid \phi\rangle \geq|\langle\psi \mid \phi\rangle|^{2}
$$

com igualdade ocorrendo se e somente se $|\psi\rangle$ e $|\phi\rangle$ são colineares. Lembramos que a função produto interno entre dois vetores $|\psi\rangle$ e $|\phi\rangle$ é definida por: $\langle\psi \mid \phi\rangle=|\psi\rangle^{\dagger}|\phi\rangle$, onde $x^{\dagger}$ denota o transposto conjugado do vetor (ou matriz) $x$. Observamos que uma maneira simples de provar a DCS é através da positividade da norma,

$$
\||\xi\rangle \|=\sqrt{\langle\xi \mid \xi\rangle} \geq 0
$$

aplicada ao vetor $|\xi\rangle=|\psi\rangle-(\langle\phi \mid \psi\rangle /\langle\phi \mid \phi\rangle)|\phi\rangle$. A condição para igualdade na DCS pode ser inferida do fato de que $\||\xi\rangle \|=0$ se e somente se $|\xi\rangle$ é o vetor nulo.

Vejamos como a DCS pode ser aplicada para a obtenção da relação de incerteza de Heisenberg, Robertson e Schrödinger (RIHRS). Consideremos $\hat{A}$ e $\hat{B}$ dois observáveis quaisquer de um sistema físico preparado no estado $|\xi\rangle$. Vamos utilizar $\langle\hat{X}\rangle=\langle\xi|\hat{X}| \xi\rangle$ para denotar o valor médio de qualquer operador $\hat{X}$ e $\mathbb{I}$ para o operador identidade em $\mathcal{H}$. Definiremos

$$
|\psi\rangle=(\hat{A}-\langle\hat{A}\rangle \mathbb{I})|\xi\rangle \text { e }|\phi\rangle=(\hat{B}-\langle\hat{B}\rangle \mathbb{I})|\xi\rangle
$$

e os substituiremos na DCS. Primeiramente notamos que

$$
\langle\psi \mid \psi\rangle=\operatorname{Var}(\hat{A}) \text { e }\langle\phi \mid \phi\rangle=\operatorname{Var}(\hat{B}),
$$

com $\operatorname{Var}(\hat{X})=\left\langle(\hat{X}-\langle\hat{X}\rangle \mathbb{I})^{2}\right\rangle$ sendo a variância de $\hat{X}$. Verificamos ainda que

$$
\begin{aligned}
\langle\psi \mid \phi\rangle & =\langle\hat{A} \hat{B}-\langle\hat{A}\rangle\langle\hat{B}\rangle \mathbb{I}\rangle \\
& =2^{-1}\langle\{\hat{A}, \hat{B}\}-2\langle\hat{A}\rangle\langle\hat{B}\rangle \mathbb{I}\rangle+2^{-1}\langle[\hat{A}, \hat{B}]\rangle,
\end{aligned}
$$

onde

$$
[\hat{A}, \hat{B}]=\hat{A} \hat{B}-\hat{B} \hat{A} \text { e }\{\hat{A}, \hat{B}\}=\hat{A} \hat{B}+\hat{B} \hat{A}
$$

são, respectivamente, o comutador e anticomutador de $\hat{A}$ com $\hat{B}$. Como $\{\hat{A}, \hat{B}\}$ e $[\hat{A}, \hat{B}]$ são, respectivamente, operadores Hermitianos e anti-Hermitianos, seus valores 
médios são, respectivamente, números reais e imaginários puros. Com isso, considerando que

$$
|\langle\psi \mid \phi\rangle|^{2}=(\operatorname{Re}\langle\psi \mid \phi\rangle)^{2}+(\operatorname{Im}\langle\psi \mid \phi\rangle)^{2},
$$

depois de algumas manipulações a DCS nos leva à RIHRS $11-\left.13\right|^{1}$

$$
\operatorname{Var}(\hat{A}) \operatorname{Var}(\hat{B}) \geq(\operatorname{CovQ}(\hat{A}, \hat{B}))^{2}+2^{-2}|\langle[\hat{A}, \hat{B}]\rangle|^{2}=T_{1},
$$

onde $\operatorname{Cov} Q(\hat{A}, \hat{B})=2^{-1}(\operatorname{Cov}(\hat{A}, \hat{B})+\operatorname{Cov}(\hat{B}, \hat{A})) \operatorname{com}$ $\operatorname{Cov}(\hat{X}, \hat{Y})=\langle\hat{X} \hat{Y}\rangle-\langle\hat{X}\rangle\langle\hat{Y}\rangle$ sendo a covariância entre os observáveis $\hat{X}$ e $\hat{Y}$.

Vamos considerar agora o problema da trivialidade da RIHRS. Sem perda de generalidade, vamos supor que o sistema foi preparado em um estado que coincide com um autovetor de $\hat{A}$, ou seja, $|\xi\rangle=\left|a_{j}\right\rangle \operatorname{com} \hat{A}\left|a_{j}\right\rangle=a_{j}\left|a_{j}\right\rangle$ e $a_{j} \in \mathbb{R}$. Nesse caso não é difícil verificar que $\operatorname{Var}(\hat{A})=$ $\operatorname{CovQ}(\hat{A}, \hat{B})=\langle[\hat{A}, \hat{B}]\rangle=0$. Com isso, a RIHRS fica

$$
0 \operatorname{Var}(\hat{B}) \geq 0,
$$

e não nos fornece informação alguma sobre a possível incompatibilidade entre os observáveis $\hat{A}$ e $\hat{B}$. Na próxima seção apresentaremos a prova de uma relação de incerteza que evita o problema da trivialidade, testemunhando a incompatibilidade entre dois observáveis mesmo quando o sistema for preparado em um de seus autovetores.

\section{A Relação de incerteza de Maccone-Pati}

Em contraste com a RIHRS, a relação de incerteza de Maccone-Pati (RIMP), que provaremos nesta seção, limita a soma das variâncias dos observáveis [14:

$$
\operatorname{Var}(\hat{A})+\operatorname{Var}(\hat{B}) \geq \max \left(L_{1}, L_{2}\right),
$$

com

$$
\begin{aligned}
& L_{1}=2^{-1}\left|\left\langle\xi|(\hat{A} \pm \hat{B})| \xi_{\perp}\right\rangle\right|^{2}, \\
& L_{2}= \pm i\langle[\hat{A}, \hat{B}]\rangle+\left|\left\langle\xi|(\hat{A} \pm i \hat{B})| \xi_{\perp}\right\rangle\right|^{2},
\end{aligned}
$$

onde $\left|\xi_{\perp}\right\rangle$ é qualquer vetor normalizado ortogonal a $|\xi\rangle$. Os sinais nas Eqs. 111) e (12) são escolhidos, respectivamente, para maximizar $L_{1}$ e $L_{2}$. Claro, como a RIMP vale para qualquer $\left|\xi_{\perp}\right\rangle$, buscaremos o vetor que leve ao maior limite inferior para a soma das variâncias. É importante notar que os limites inferiores $L_{1}$ e $L_{2}$ serão nulos somente se o estado do sistema, $|\xi\rangle$, for um autovetor comum dos dois observáveis $\hat{A}$ e $\hat{B}$. Vale mencionar que a RIMP já foi verificada experimentalmente no caso especial de observáveis representados por operadores Hermitianos e unitários 38].

${ }^{1}$ Embora essa desigualdade seja comumente chamada de relação de incerteza de Heisenberg, aqui preferimos atribuí-la também a Robertson e Schrödinger, que a obtiveram de forma mais geral. Uma demonstração alternativa da RIHRS pode ser encontrada na Ref. 37.
Vale enfatizar também que a novidade na RIMP não é simplesmente a utilização da soma das variâncias em lugar do produto. Podemos facilmente obter uma RIHRS envolvendo soma de variâncias partindo de $\left(\sigma_{A}-\sigma_{B}\right)^{2} \geq$ 0 , com o desvio padrão do observável $\hat{X}$ sendo definido como $\sigma_{X}=\sqrt{\operatorname{Var}(\hat{X})}$. Essa desigualdade implica que

$$
\operatorname{Var}(\hat{A})+\operatorname{Var}(\hat{B}) \geq 2 \sigma_{A} \sigma_{B} \geq|\langle[\hat{A}, \hat{B}]\rangle|=T_{2},
$$

com a última desigualdade sendo um caso particular da RIHRS, Eq. (8). No entanto, pode-se verificar que se o estado no qual o sistema foi preparado for um autovetor de um dos observáveis, a relação de incerteza na Eq. (13) também apresenta o problema da trivialidade.

\subsection{Prova do primeiro limite na RIMP}

Para provar a RIMP, faremos uso da lei dos paralelogramos, que está ilustrada na Fig. 1 e estabelece que, para quaisquer dois vetores $|\psi\rangle$ e $|\phi\rangle$ no espaço de Hilbert $\mathcal{H}$, a seguinte igualdade é verdadeira:

$$
2\left(\||\psi\rangle\left\|^{2}+\right\||\phi\rangle \|^{2}\right)=\|(|\psi\rangle+|\phi\rangle)\left\|^{2}+\right\|(|\psi\rangle-|\phi\rangle) \|^{2} .
$$

Vamos inserir os vetores definidos na Eq. (3) na lei dos paralelogramos, Eq. 14. Como $\||\psi\rangle \|^{2}=\operatorname{Var}(\hat{A}) \mathrm{e}$ $\||\phi\rangle \|^{2}=\operatorname{Var}(\hat{B})$ teremos

$$
\begin{aligned}
& \operatorname{Var}(\hat{A})+\operatorname{Var}(\hat{B}) \\
& =2^{-1}\left(\|(|\psi\rangle+|\phi\rangle)\left\|^{2}+\right\|(|\psi\rangle-|\phi\rangle) \|^{2}\right) \\
& \geq 2^{-1} \|(|\psi\rangle \pm|\phi\rangle) \|^{2} \\
& =2^{-1}\left(\left\langle\psi| \pm\langle\phi|)(|\psi\rangle \pm|\phi\rangle)\left\langle\xi_{\perp} \mid \xi_{\perp}\right\rangle\right.\right. \\
& \geq 2^{-1} \mid\left(\left.\left\langle\psi| \pm\langle\phi|) \mid \xi_{\perp}\right\rangle\right|^{2}\right. \\
& =2^{-1}\left|\left\langle\xi|(\hat{A} \pm \hat{B})| \xi_{\perp}\right\rangle-(\langle\hat{A}\rangle \pm\langle\hat{B}\rangle)\left\langle\xi \mid \xi_{\perp}\right\rangle\right|^{2} \\
& =2^{-1}\left|\left\langle\xi|(\hat{A} \pm \hat{B})| \xi_{\perp}\right\rangle\right|^{2}=L_{1} .
\end{aligned}
$$

Obtivemos a desigualdade na Eq. 16 a partir da igualdade na Eq. (15) utilizando a positividade da norma. Passamos de (16) pra (17) e de (19) pra (20) usando um vetor $\left|\xi_{\perp}\right\rangle$ normalizado e ortogonal ao estado do sistema $|\xi\rangle$. Já a desigualdade na Eq. (18) é consequência da desigualdade de Cauchy-Schwarz, Eq. (1). O sinal

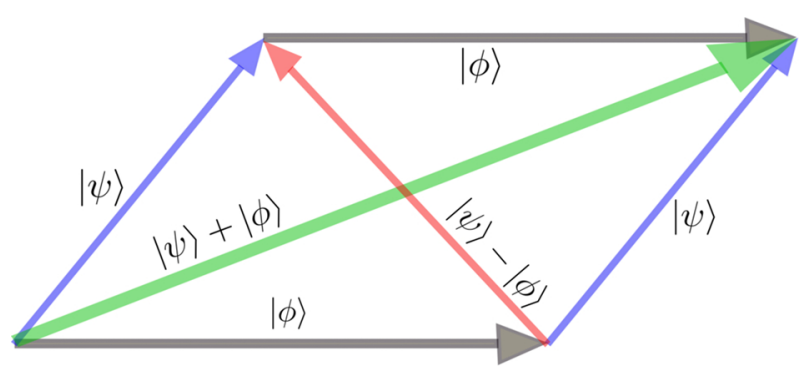

Figura 1: Lei dos paralelogramos: A soma dos quadrados das diagonais de um paralelogramo é igual à soma dos quadrados dos seus lados. Em termos das normas dos vetores correspondentes, essa lei se traduz na Eq. (14). 
nas equações acima indica se usaremos $\|(|\psi\rangle+|\phi\rangle) \|^{2}$ ou $\|(|\psi\rangle-|\phi\rangle) \|^{2}$ ao passarmos de 15) pra (16) e é escolhido de forma a maximizar $L_{1}$.

\subsection{Prova do segundo limite na RIMP}

Aplicando os mesmos procedimentos da última sub-seção, podemos verificar que

$$
\begin{aligned}
\|(|\psi\rangle \pm i|\phi\rangle) \|^{2} & =(\langle\psi|\mp i\langle\phi|)(|\psi\rangle \pm i|\phi\rangle) \\
& =\||\psi\rangle\left\|^{2}+\right\||\phi\rangle \|^{2} \pm i(\langle\psi \mid \phi\rangle-\langle\phi \mid \psi\rangle) \\
& =\|\psi \psi\|^{2}+\||\phi\rangle \|^{2} \pm i\langle[\hat{A}, \hat{B}]\rangle \quad(21)
\end{aligned}
$$

e

$$
\begin{aligned}
\|(|\psi\rangle \pm i|\phi\rangle) \|^{2}= & \left(\left\langle\psi|\mp i\langle\phi|)(|\psi\rangle \pm i|\phi\rangle)\left\langle\xi_{\perp} \mid \xi_{\perp}\right\rangle\right.\right. \\
\geq & \mid\left(\left.\left\langle\psi|\mp i\langle\phi|) \mid \xi_{\perp}\right\rangle\right|^{2}\right. \\
= & \mid\left\langle\xi|(\hat{A} \mp i \hat{B})| \xi_{\perp}\right\rangle-(\langle\hat{A}\rangle \\
& \mp i\langle\hat{B}\rangle)\left.\left\langle\xi \mid \xi_{\perp}\right\rangle\right|^{2} \\
= & \left|\left\langle\xi|(\hat{A} \mp i \hat{B})| \xi_{\perp}\right\rangle\right|^{2} .
\end{aligned}
$$

Assim, se utilizamos $i|\phi\rangle$ em lugar do $|\phi\rangle$ na lei dos paralelogramos, como $\| i|\phi\rangle\|=\||\phi\rangle \|$, obteremos

$$
\begin{aligned}
2(\operatorname{Var}(\hat{A})+\operatorname{Var}(\hat{B})) \geq & \operatorname{Var}(\hat{A})+\operatorname{Var}(\hat{B}) \pm i\langle[\hat{A}, \hat{B}]\rangle \\
& +\left|\left\langle\xi|(\hat{A} \pm i \hat{B})| \xi_{\perp}\right\rangle\right|^{2}, \quad(23)
\end{aligned}
$$

da qual prontamente obtemos o limite $L_{2}$ da Eq. (12). O sinal na Eq. (23), que está relacionado a qual dos termos $\|(|\psi\rangle \pm i|\phi\rangle) \|^{2}$ na Eq. (14) aplicaremos a desigualdade (22), é escolhido de modo a maximizar $L_{2}$.

\section{Exemplo: Complementaridade para um qubit}

Nesta seção, vamos olhar para um sistema de dois níveis, um qubit, preparado no estado

$$
|\xi\rangle=2^{-1 / 2}\left(|0\rangle+e^{i \alpha}|1\rangle\right),
$$

com $|0\rangle$ e $|1\rangle$ sendo os autovetores da matriz de Pauli $\hat{Z}=|0\rangle\langle 0|-| 1\rangle\langle 1|$ e $\alpha \in[0,2 \pi)$. Claro, tudo que será dito nesta sub-seção vale para o popular exemplo da partícula de spin 1/2 medida via aparatos de SternGerlach 8. Consideraremos a aplicação das RIHRS e RIMP para indicar a bem conhecida incompatibilidade entre os observáveis $\hat{Z}$ e $\hat{X}=|0\rangle\langle 1|+| 1\rangle\langle 0|$. Pode-se verificar que para o estado preparado: $\langle\hat{Z}\rangle=0,\langle\hat{X}\rangle=$ $\cos \alpha$ e $\langle\hat{Z} \hat{X}\rangle=-\langle\hat{X} \hat{Z}\rangle=i \sin \alpha$. Com isso temos que $\operatorname{Cov} Q(\hat{X}, \hat{Z})=0$ e $|\langle[\hat{X}, \hat{Z}]\rangle|^{2}=2^{2} \sin ^{2} \alpha$. Os dois limites inferiores nas RIHRS das Eqs. (8) e (13) são então dados por

$$
T_{1}=2^{-2} T_{2}^{2}=\sin ^{2} \alpha .
$$

Levando em conta que neste exemplo existe um único vetor normalizado ortogonal a $|\xi\rangle:\left|\xi_{\perp}\right\rangle=2^{-1 / 2}(|0\rangle-$ $\left.e^{i \alpha}|1\rangle\right)$, depois de alguns cálculos simples, obtemos os limites impostos pela RIMP, Eqs. (11) e (12):

$$
L_{2}=2 L_{1}=1+\sin ^{2} \alpha .
$$

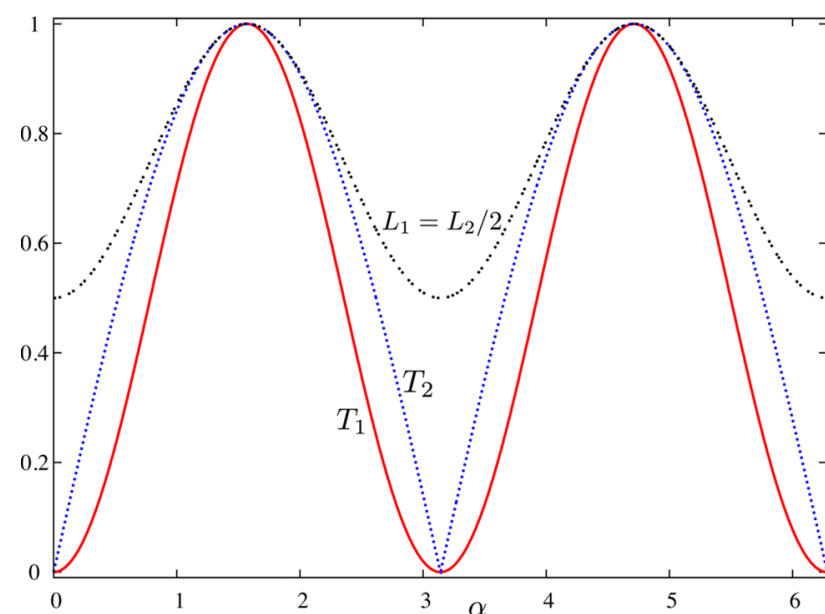

Figura 2: Limites para as variâncias dos observáveis $\hat{X}$ e $\hat{Z}$ impostos pelas relações de incerteza de Heisenberg-RobertsonSchrödinger $\left(T_{1}\right.$ e $\left.T_{2}\right)$ e de Maccone-Pati $\left(L_{1}\right.$ e $\left.L_{2}\right)$ para um qubit preparado no estado $|\xi\rangle=2^{-1 / 2}\left(|0\rangle+e^{i \alpha}|1\rangle\right)$.

Esses quatro limites para as variâncias de $\hat{X}$ e $\hat{Z}$ estão mostrados na Fig. 22 Vemos que embora o comportamento qualitativo geral das curvas seja parecido, existem importantes diferenças quantitativas para as fases $\alpha=\{0, \pi, 2 \pi\}$. Para esses valores de $\alpha$, o estado no qual o sistema foi preparado, $|\xi\rangle$, é um autovetor de $\hat{X}$ e, em contraste com a RIMP, a RIHRS, devido ao problema da trivialidade, não é capaz de indicar que a largura da distribuição de probabilidades para os autovalores de $\hat{Z}$ não é nula.

\section{Considerações finais}

Neste artigo, depois de discutir brevemente alguns aspectos do princípio de incerteza da Mecânica Quântica (MQ), apresentamos uma prova didática da relação de incerteza de Maccone-Pati (RIMP) e exemplificamos sua aplicação em um sistema de dois níveis. É um fato curioso que uma restrição tão importante dentro da $\mathrm{MQ}$ como a RIHRS tenha um problema relevante que, embora provavelmente tenha sido notado por vários professores e pesquisadores da área, foi resolvido tanto tempo após sua concep̧̧ão. Esperamos portanto que a demonstração simples da RIMP que apresentamos neste artigo sirva de motivação para que ela seja prontamente incluída nas disciplinas de MQ de cursos de graduação e de pós graduação em Física e áreas correlatas.

\section{Agradecimentos}

Este trabalho foi financiado pelo $\mathrm{CNPq}$, processos 441875/2014-9 e 303496/2014-2, pelo Instituto Nacional de Ciência e Tecnologia de Informação Quântica (INCTIQ), processo 2008/57856-6, e pela CAPES, processo 6531/2014-08. 


\section{Referências}

[1] L. Mlodinow, The Drunkard's Walk: How Randomness Rules our Lives (Pantheon Books, Nova York, 2008).

[2] M. Bell, K. Gottfried and M. Veltman, John Bell on The Foundations of Quantum Mechanics (World Scientific, Singapura, 2001).

[3] A. Aspect, Physics 8, 123 (2015).

[4] B. Hensen, H. Bernien, A.E. Dréau, A. Reiserer, N. Kalb, M.S. Blok, J. Ruitenberg, R.F.L. Vermeulen, R.N. Schouten, C. Abellán, et al., Nature 526, 682 (2015).

[5] M. Giustina, M.A.M. Versteegh, S. Wengerowsky, J. Handsteiner, A. Hochrainer, K. Phelan, F. Steinlechner, J. Kofler, J.-Å. Larsson, C. Abellán, W. Amaya et al., Phys. Rev. Lett. 115, 250401 (2015).

[6] L.K. Shalm, E. Meyer-Scott, B.G. Christensen, P. Bierhorst, M.A. Wayne, M.J. Stevens, T. Gerrits, S. Glancy, D.R. Hamel, M.S. Allman, et al., Phys. Rev. Lett. 115, 250402 (2015).

[7] D.J. Griffiths, Mecânica Quântica (Pearson Education, São Paulo, 2011).

[8] J.J. Sakurai e J. Napolitano, Mecânica Quântica Moderna (Bookman, Porto Alegre, 2013).

[9] J. Maziero, Rev. Bras. Ensino Fís. 37, 1314 (2015).

[10] J. Maziero, Rev. Bras. Ensino Fís. 38, e2307 (2016).

[11] W. Heisenberg, Zeitschrift für Physic 43, 172 (1927).

[12] H.P. Robertson, Phys. Rev. 34, 163 (1929).

[13] E. Schrödinger, Proceedings of the Prussian Academy of Sciences, Physics-Mathematical Section 14, 296 (1930). Tradução para o inglês, por A. Angelow e M.-C. Batoni, disponível em arXiv: quant-ph/9903100

[14] L. Maccone and A.K. Pati, Phys. Rev. Lett. 113, 260401 (2014).

[15] P.J. Lahti and M.J. Maczynski, J. Math. Phys. 28, 1764 (1987).

[16] M. Koashi, J. Phys.: Conf. Ser. 36, 98 (2006).

[17] H.F. Hofmann and S. Takeuchi, Phys. Rev. A 68, 032103 (2003).

[18] O. Gühne, Phys. Rev. Lett. 92, 117903 (2004).

[19] P. Busch, T. Heinonen and P. Lahti, Phys. Rep. 452, 155 (2007).

[20] M.A. Nielsen and I.L. Chuang, Quantum Computation and Quantum Information (Cambridge University Press, Cambridge, 2000).

[21] M.M. Wilde, Quantum Information Theory (Cambridge University Press, Cambridge, 2013).

[22] M. Ozawa, Phys. Lett. A 320, 367 (2004).

[23] C. Branciard, PNAS 110, 6742 (2013).

[24] F. Buscemi, M.J.W. Hall, M. Ozawa and M.M. Wilde, Phys. Rev. Lett. 112, 050401 (2014).

[25] R. Horodecki, P. Horodecki, M. Horodecki and K. Horodecki, Rev. Mod. Phys. 81, 865 (2009).

[26] L. Aolita, F. de Melo and L. Davidovich, Rep. Prog. Phys. 78, 042001 (2015).

[27] L.C. Céleri, J. Maziero and R.M. Serra, Int. J. Quantum Inf. 09, 1837 (2011).

[28] K. Modi, A. Brodutch, H. Cable, T. Paterek and V. Vedral, Rev. Mod. Phys. 84, 1655 (2012).

[29] M. Berta, M. Christandl, R. Colbeck, J.M. Renes and R. Renner, Nat. Phys. 6, 659 (2010).

[30] A.K. Pati, M.M. Wilde, A.R.U. Devi, A.K. Rajagopal and Sudha, Phys. Rev. A 86, 042105 (2012).
[31] S. Wehner and A. Winter, New J. Phys. 12, 025009 (2010).

[32] S.L. Braunstein, C.M. Caves and G.J. Milburn, Ann. Phys. 247, 135 (1996).

[33] M.R. Frey, Quantum Inf. Process. 15, 3919 (2016).

[34] P. Busch and N. Stevens, Phys. Rev. Lett. 114, 070402 (2015).

[35] F. Buscemi, M. Dall'Arno, M. Ozawa and V. Vedral, arXiv: 1312.4240

[36] F. Buscemi, M. Dall'Arno, M. Ozawa and V. Vedral, Int. J. Quantum Inf. 12, 1560002 (2014).

[37] G. Rigolin, Eur. J. Phys. 36, 065007 (2015).

[38] K. Wang, X. Zhan, Z. Bian, J. Li, Y. Zhang and P. Xue, Phys. Rev. A 93, 052108 (2016). 